\title{
Stationary-phase slip state in quasi-one-dimensional rings
}

\author{
D. Y. Vodolazov, B. J. Baelus, and F. M. Peeters* \\ Departement Natuurkunde, Universiteit Antwerpen (UIA), Universiteitsplein 1, B-2610 Antwerpen, Belgium
}

(Received 15 February 2002; published 22 August 2002)

\begin{abstract}
The nonuniform superconducting state in a ring in which the order parameter vanishing at one point is studied. This state is characterized by a jump of the phase by $\pi$ at the point where the order parameter becomes zero. In uniform rings such a state is a saddle-point state and consequently unstable. However, for nonuniform rings with, e.g., variations of geometrical or physical parameters or with attached wires this state can be stabilized and may be realized experimentally.
\end{abstract}

DOI: $10.1103 /$ PhysRevB.66.054531

PACS number(s): 74.60.Ec, 74.20.De, 73.23.-b

In recent years ${ }^{1-3}$ the existence of a single-connected state in a ring was proposed theoretically. Such a state implies that the relation between the phase $\phi$ of the order parameter $\psi$ $=f e^{\mathrm{i} \phi}$, the current density $j$, and the magnetic flux $\Phi$ through the ring (which follows from the single valuedness of $\psi$-see, for example, Ref. 4),

$$
\oint \frac{j}{f^{2}} d s=2 \pi n-\Phi
$$

is no longer valid (the flux is expressed in $\Phi_{0} / 2 \pi$, the current density in $j_{0}=c \Phi_{0} / 4 \pi^{2} \lambda^{2} \xi$, $\lambda$ is the London penetration length, $\xi$ the coherence length, and $\Phi_{0}$ is the quantum of magnetic flux). The reason is that the order parameter in such a single-connected state is zero at one point. Moreover, it was claimed that under certain conditions, i.e., radius of the ring less than $\xi$ and the flux through the ring is about ( $n$ $+1 / 2) \Phi_{0}$, this state may become metastable in some range of magnetic fields. ${ }^{2}$

In this paper we revisited this problem and we will show that a state where the order parameter vanishes in one point is still double connected. By this we mean that the phase of the order parameter is not independent at both sides of the point where $\psi=0$, and Eq. (1) remains valid when the limit of $j \rightarrow 0$ is properly taken. We propose to call such a state a one-dimensional (1D) vortex state (ODV state), because like for an ordinary two-dimensional Abrikosov vortex, there is a jump in the phase of the order parameter of $\pi$ at the point where $\psi=0$. In contrast to a two-dimensional Abrikosov vortex the $1 \mathrm{D}$ vortex is an unstable structure in a uniform ring. However, if some inhomogeneities are present in the ring (defects, nonuniform thickness or nonuniform width of the ring, attached superconducting wires) the ODV structure can be stabilized and may consequently be realized experimentally.

Consider a uniform ring with thickness $d \lesssim \lambda$ and width $w \lesssim \xi$. In addition, let the radius of the ring $R$ be much larger than $w$. Under these conditions we can neglect the screening effects and the problem is reduced to a one-dimensional one. The distribution of the current density $j$ and the order parameter $\psi$ of the system at a temperature not far from the critical temperature $T_{c}$ is described by the 1D Ginzburg-Landau equation (plus the condition $\operatorname{div} \mathbf{j}=0$ ),

$$
\begin{gathered}
\frac{d^{2} f}{d s^{2}}+f\left(1-f^{2}-p^{2}\right)=0, \\
\frac{d j}{d s}=\frac{d}{d s} f^{2} p=0,
\end{gathered}
$$

where the gauge-invariant momentum $p=\nabla \phi-A$ is scaled in units of $\Phi_{0} /(2 \pi \xi)$, the length of the ring is $L=2 \pi R$ and the circular coordinate $s$ is in units of the coherence length $\xi$. In these units, the magnetic field is scaled in units of the second critical field $H_{c 2}$ and the magnetic flux in $\Phi_{0} / 2 \pi$.

The coupled Eqs. (2a) and (2b) have to be solved with the boundary condition $\psi(-L / 2)=\psi(L / 2)$. We use the method proposed in Ref. 5 (see also Ref. 6). These equations have the first integral,

$$
\frac{1}{2}\left(\frac{d f}{d s}\right)^{2}+\frac{f^{2}}{2}-\frac{f^{4}}{4}+\frac{j^{2}}{2 f^{2}}=E .
$$

From a formal point of view, Eq. (3) is nothing else than the condition of "energy" $(E)$, conservation for some "particle" with "coordinate" $f$ and "momentum" $j$. The role of "time" is played ${ }^{5}$ by the circular coordinate $s$. In Fig. 1 we show the dependence of the "potential energy" of this system,

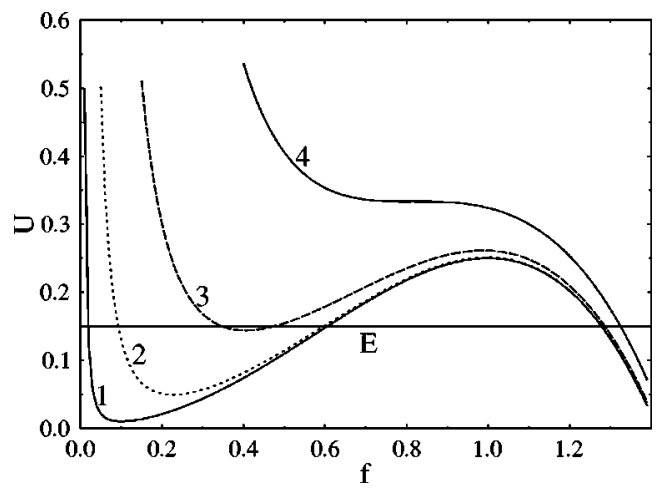

FIG. 1. Dependence of the potential energy $U(f)$ for different values of the current $j .1: j=0.01 ; 2: j=0.05 ; 3: j=0.15$; 4: $j=j_{c}=\sqrt{4 / 27}$. 


$$
U(f)=\frac{f^{2}}{2}-\frac{f^{4}}{4}+\frac{j^{2}}{2 f^{2}},
$$

for different values of $j$. Possible solutions of Eqs. (2a) and (2b) are in the region where confined "trajectories" of our virtual particle exist. This is possible for currents $0<j \leqslant j_{c}$ ( $j_{c}=\sqrt{4 / 27} j_{0}$ is the depairing current density).

Using Eq. (3) we immediately can write the solution of Eqs. (2a) and (2b) for the ring

$$
\begin{aligned}
\sqrt{2} s & =\int_{t_{0}}^{t} \frac{d t}{\sqrt{\left(t-t_{0}\right)\left(t_{1}-t_{0}\right)\left(t_{2}-t_{0}\right)}} \\
& =\frac{2}{\sqrt{t_{2}-t_{0}}} F\left(\sin ^{-1} \sqrt{\frac{t-t_{0}}{t_{1}-t_{0}}}, \sqrt{\frac{t_{1}-t_{0}}{t_{2}-t_{0}}}\right),
\end{aligned}
$$

where $t(s)=f^{2}(s), F(\theta, m)$ is the elliptic integral of the first kind, and $t_{0} \leqslant t_{1} \leqslant t_{2}$ are the solutions of the cubic equation

$$
t^{3}-2 t^{2}+4 E t-2 j^{2}=0 .
$$

Using the boundary condition for $f$ we may conclude that for a given current there exists a maximum of three solutions. There are two uniform solutions [in the points of the minimum $E=E_{\text {min }}$ and the maximum $E=E_{\text {max }}$ of the potential energy (4)] and one nonuniform solution with energy $E_{\min }$ $<E<E_{\max }$ which is defined by the equation

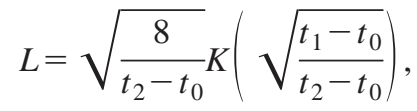

where $K(m)$ is the complete elliptic integral of the first kind. Numerical analysis of Eq. (7) for $0<j<j_{c}$ shows that there is a minimal value for the ring length $L_{\min }$ for which there exists a solution for this equation. When $j \rightarrow j_{c}, L_{\min } \rightarrow \infty$ and in the opposite limit $j \rightarrow 0$ one can show that $L_{\min } \rightarrow \pi$. The latter corresponds to a ring radius $R=1 / 2$ (or $\xi / 2$ in dimensional units). For a radius $R>1 / 2$ metastable states may exist and superconductivity is present for any value of the magnetic flux (at least in our one-dimensional model). ${ }^{2,7}$

In principle, Eqs. (5)-(7) define the nonuniform solution of Eqs. (2a) and (2b) for a ring. Unfortunately, even using the explicit Kordano expressions for the roots of Eq. (6) the results are rather complicated for arbitrary values of the current $j$. However, a tractable analytical solution is possible if we consider the case of low currents $j \ll 1$ for which the roots of Eq. (6) are simplified to

$$
\begin{gathered}
t_{0} \simeq j^{2} / 2 E, \\
t_{1} \simeq 1-\sqrt{1-4 E}, \\
t_{2} \simeq 1+\sqrt{1-4 E} .
\end{gathered}
$$

After inserting these results into Eq. (7) we obtain the energy. For $L \gg \pi$ the energy $E \simeq 1 / 4$ is practically independent of $L$ (e.g., for $L=8$ the difference $1 / 4-E(L=8)$ is less than $0.002)$. Also in the limit $L-\pi \ll 1$ we found that the energy $E$ is independent of $j$,

$$
E(L) \simeq \frac{1}{2}\left(\frac{L-\pi}{\pi / 8}\right)^{2}
$$

In the limit $j \ll 1$ we can also find ${ }^{8}$ the dependence of $t(s)$ near the minimum point of $t(s)$,

$$
\begin{gathered}
t(s)=2 j^{2}+s^{2} / 2, \quad L \gg \pi, \quad s \ll 1, \\
t(s)=j^{2} / 2 E+2 E s^{2}, \quad L-\pi \ll 1, \quad s \ll 1 .
\end{gathered}
$$

Using Eqs. (10a) and (10b) it is easy to show that in the limit $j \rightarrow 0$ the gauge-invariant momentum is given by

$$
p(s)=j / t(s)=\frac{j}{|j|} \pi \delta(s),
$$

where $\delta(s)$ is the Dirac function. As a result the integral

$$
\begin{aligned}
\lim _{j \rightarrow 0} \oint \frac{j}{f^{2}} d s & =\lim _{j \rightarrow 0} \oint p(s) d s \\
& =\phi(+\epsilon)-\phi(-\epsilon) \\
& =\Delta \phi= \pm \pi,
\end{aligned}
$$

for arbitrary ring size. ${ }^{9}$ Combining Eq. (12) with Eq. (1) it is easy to show that if $\Phi=(k+1 / 2) \Phi_{0}$ ( $k$ is an integer) a solution of Eq. (2a) can be found which vanishes at one point.

In previous ${ }^{1-3}$ studies of this state Eq. (11) was not taken into account (only the absolute value of the order parameter was found). Furthermore, in Ref. 2 it was claimed that for small rings there is a magnetic-field region in which such a state exists and is metastable. But we find that in this region this state does not even exist (except in one point) because Eq. (1) is not fulfilled!

The distribution of the order parameter as obtained in the present paper may also be applied to a ring in which some part of the ring consists of a normal metal. Then at the boundary between the normal metal and the superconductor the condition $\psi \simeq 0$ is fulfilled (if the normal part is longer than $\xi)$ and the distribution of the density $|\psi(s)|^{2}$ coincides with the one obtained in Ref. 2 [but the phase $\phi(s)$ will be different for our previous ring geometry]. Besides we cannot call such a state single connected as in Refs. 1-3 because the relation (1) is valid even for this case. Therefore it is better to call this state a one-dimensional vortex state because like the two-dimensional Abrikosov vortex there is a point where $|\psi|=0$ and the phase of the order parameter exhibits a jump equal to $\pi$ (as the Abrikosov vortex with orbital momentum $2 \pi)$.

Our numerical analysis of the time-dependent GinzburgLandau equations showed that this state is completely unstable for a uniform ring. However, for rings with nonuniform width (thickness) or attached wires a nonuniform distribution of the order parameter becomes possible $e^{1,3,10,11}$ and for small rings with $L \sim \pi$ this state is realized in practice (besides for the case of a ring, a ODV state can also exist in the Wheatstone bridge-see Refs. 12 and 13). In Fig. 2 the distribution of the absolute value and the phase of the order parameter is shown for different values of the magnetic field 


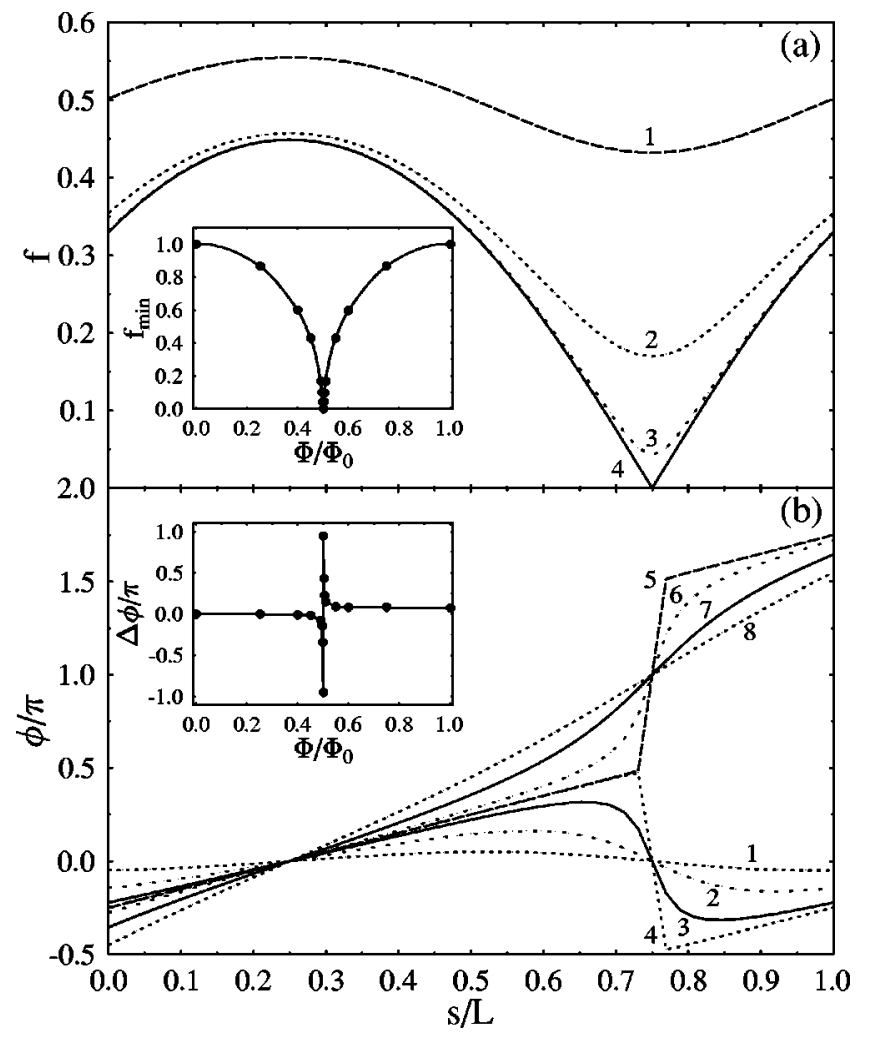

FIG. 2. Distribution of the absolute value (a) and phase (b) of the order parameter in a nonuniform ring for different values of the magnetic flux. 1: $\Phi=0.45 \Phi_{0} ; 2: \Phi=0.49 \Phi_{0} ; 3: \Phi=0.498 \Phi_{0} ; 4$ : $\Phi=(0.5-0) \Phi_{0} ; \quad 5: \quad \Phi=(0.5+0) \Phi_{0} ; \quad 6: \quad \Phi=0.502 \Phi_{0} ; \quad 7: \quad \Phi$ $=0.51 \Phi_{0} ; 8: \Phi=0.55 \Phi_{0}$. Note that the order parameter for $\Phi$ $=\Phi_{0} / 2+\alpha$ is the same as for $\Phi=\Phi_{0} / 2-\alpha$ when $\alpha<\Phi_{0} / 2$. In the insets (a) $f_{\min }$ and (b) the phase difference $\Delta \phi$ near the point $s / L$ $=3 / 4$ are shown as function of $\Phi$.

for a nonuniform ring. ${ }^{14}$ We used the following model where the width of the ring was varied as

$$
w(s)=1+w_{0} \sin \left(\frac{2 \pi s}{L}\right) .
$$

The parameters used are $w_{0}=0.1$ and $L=3.25$. It is seen that with increasing magnetic flux the magnitude of the order parameter $f$ decreases in the thinner part of the ring (at $s / L$ $=3 / 4$ ) and becomes zero when $\Phi / \Phi_{0}=1 / 2$. In Ref. 3 it was found that the ODV state is only possible at $\Phi=(n$ $+1 / 2) \Phi_{0}$. The reason is now clear-only at this value of the magnetic flux the phase difference near the point where the order parameter is zero will be compensated by the term $2 \pi n-\Phi$ and the current density will be equal to zero. It is interesting to note that the current density is also zero for $\Phi=n \Phi_{0}$ even in the case of a nonuniform ring, but for values of the flux where the ODV state does not exist. At these values of the magnetic flux the order parameter is uniform along the ring and the term $2 \pi n$ is completely compensated by the term $\Phi$ in Eq. (1).

Above we showed that the ODV state can be realized by varying the geometrical parameters of the ring. But there are two alternative approaches to realize the ODV state in the

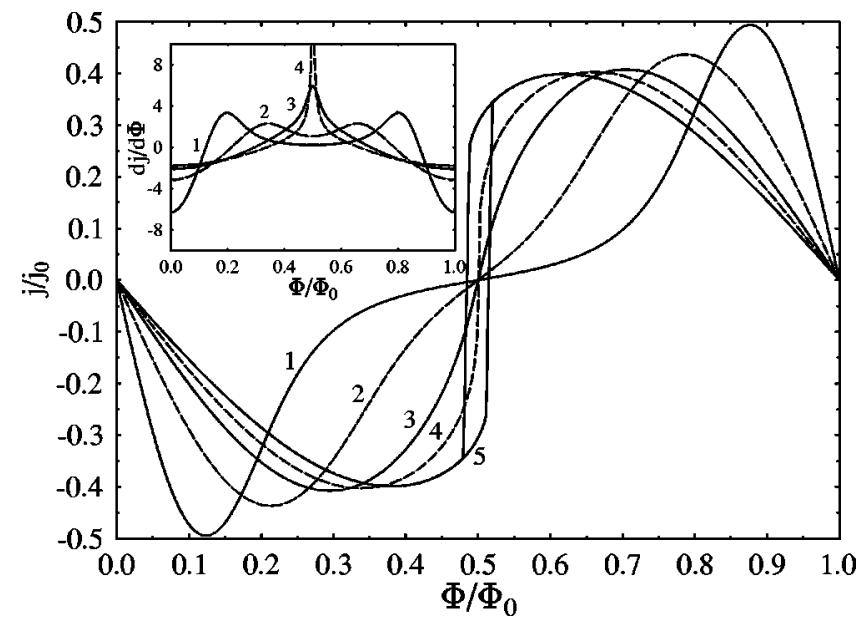

FIG. 3. The current in the ring (with attached wire of length $5 \xi$ ) as function of the applied flux. The different curves are for different circumference of the ring. $1: L=1 ; 2: L=2 ; 3: L=3 ; 4: L=3.5 ; 5$ : $L=4$. For $L=4$ the ODV state does not exist in the ring and hysteresis appears. In the inset the dependence of $d j / d \Phi$ on the flux $\Phi$ is shown for rings with lengths $L=1,2,3,3.5$ (curves $1,2,3,4$, respectively). Note that there are two maxima in the range $(0,1)$. With increasing length the two maxima merge into one and when one approaches the critical length $d j / d \Phi$ diverges at $\Phi=\Phi_{0} / 2$.

ring experimentally. First, it is possible to include another phase in the ring. This leads to the appearance of a weak link in the sample and if the radius of the ring is less than some critical value (about $\xi$ ) it also leads to the existence of the ODV state in the ring at $\Phi=(n+1 / 2) \Phi_{0}$. We modeled this situation by introducing an additional term $\rho(s) f$ in Eq. (2a), and, as an example, we took $\rho(s)=-\alpha \delta(s)$ and $\alpha=1$ (it should be noted that qualitatively the results do not depend on the specific value of $\alpha$ ). We will not present the numerical results of the modified Ginzburg-Landau equations but we found that they are qualitatively similar to the behavior of $f$ and $\phi$ shown in Fig. 2. When approaching the flux $\Phi$ $=(n+1 / 2) \Phi_{0}$ the order parameter reaches zero in the defect point and a jump in the phase equal to $\pi$ occurs. Second, such a ODV state should also appear in small rings with attached wire(s). As was shown in Refs. 10 and 11 an attached wire leads to a nonuniform distribution of the order parameter in the ring. In Fig. 3 the dependence of the current $j$ in such a ring on the flux through the ring is shown (see also Ref. 11). As for nonuniform rings, where the parameters are chosen such that the ODV state may exist, there is no hysteresis in such a system. ${ }^{3}$ In addition for rings with attached wire the dependence $d j / d \Phi$ on $\Phi$ have two maxima in the range $(0,1)$ for small $L$ in agreement with the case for nonuniform rings (see Ref. 3 ). In contrast to the nonuniform ring case superconductivity survives in a ring with attached wire near $\Phi=\Phi_{0}(n+1 / 2)$ and for $L<\pi$.

Small inhomogeneities in rings gives us the unique possibility to study states close to the ODV state because there is no sharp transition from the state with finite order parameter to a state with vanishing order parameter at one point (see Fig. 2). The latter is, in some sense, a "frozen" slip phase state. A small variation of the flux through the ring near $\Phi$ 


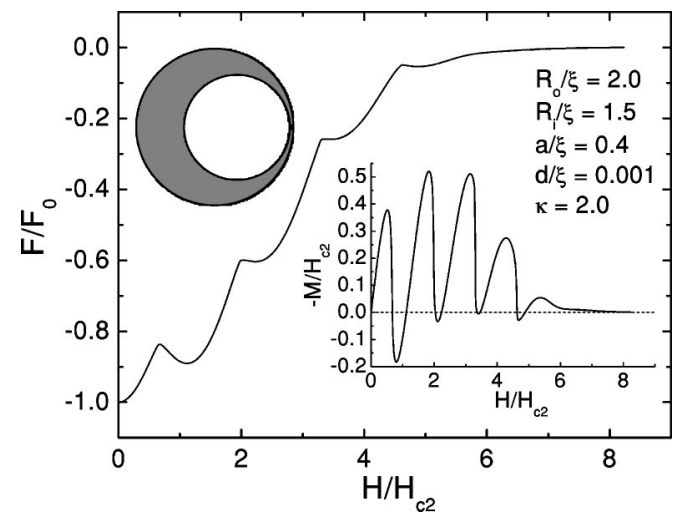

FIG. 4. The Gibbs free energy and the magnetization (right lower inset) of the asymmetric ring (upper inset) as function of the applied magnetic field.

$=\Phi_{0} / 2$ leads to a change of $\Delta \phi$ by $2 \pi$ [from $-\pi$ to $+\pi$ with a concomitant change of the current from -0 to +0 - see Fig. 2(b)]. As a result an additional phase circulation $\oint \nabla \phi d s=2 \pi$ appears in the system, not because the magnetic flux $\Phi$ is changed, but because the term $\oint j / f^{2} d s$ changes from $-\pi$ to $+\pi$ [see Eq. (1)]. When such a jump occurs $\nabla \phi$ does not change in the ring, except near the point where $f=0$, and as a result the current density in the system changes continuously. In the usual case transitions of the vorticity from a state with phase circulation $2 \pi n$ to a state with phase circulation $2 \pi(n+1)$ leads to a jump in the current everywhere in the ring because $\oint \nabla \phi d s$ changes by $2 \pi$ and the order parameter and the current density are finite everywhere in the system.

To supplement the above study we made also a numerical study of a nonuniform ring of finite width and thickness by implementing our previous finite difference solution of the coupled nonlinear Ginzburg-Landau equations. ${ }^{15}$ As an example we took the following parameters: outer radius of the ring $R_{o}=2 \xi$, radius of the hole $R_{i}=1.5 \xi$, displacement of the hole from the center $a=0.4 \xi$, ring thickness $d=0.001 \xi$, and Ginzburg-Landau parameter $\kappa=2$.

In Fig. 4 the dependence of the Gibbs free energy $F$ and the magnetization $M=-\partial F / \partial H$ of this system on the magnetic field are shown. As in the case of our one-dimensional ring these dependencies are reversible and there are magnetic fields at which the magnetization is equal to zero (in the points of local maximum and minimum of the free energy). We found that the distribution of the order parameter and the phase in the ring (see inset of Fig. 5) is similar to analogical distributions for the above one-dimensional ring (Fig. 2) at low magnetic fields. But different with the one-dimensional system the phase circulation increase of $2 \pi$ [between the points (2) and (3) in Fig. 5] does not occur at the magneticfield value where the free energy has a local maximum (and zero magnetization). With decreasing width of the ring this point shifts towards the local maximum in the free energy. It is interesting to note that for the system corresponding to Fig. 4 the vortex enters through the thinnest part of the ring in the case of the first three maxima in the free energy while for the highest magnetic field maxima (i.e., $H / H_{c 2} \simeq 4$.6) the

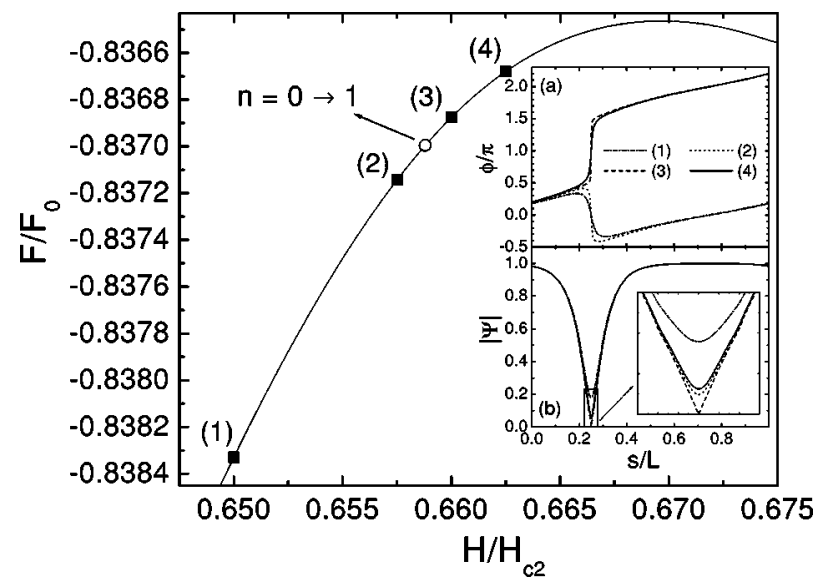

FIG. 5. The Gibbs free energy $F(H)$ near the first maximum. In the inset the phase and the order parameter distribution at different values of the applied magnetic field [indicated by the squares on the $F(H)$ curve] are shown. The phase was calculated along the outer perimeter of the ring. The open circle on $F(H)$ indicates the position at which the vorticity increases from 0 to 1 .

vortex enters through the thickest part of the ring (see Fig. $6)$. Because the width of thicker part of the ring $(=0.9 \xi)$ is considerably larger than the thinner part $(=0.1 \xi)$ and is of order $\xi$ the ODV for $H / H_{c 2} \simeq 4.6$ becomes the usual twodimensional vortex. As a result the order parameter is equal to zero only in one point along the radial coordinate and the circulation of the phase of the order parameter now is also a function of this coordinate (see Fig. 6). When the vortex enters/exits the ring at low magnetic fields there is also a slow dependence of the order parameter on the radial coordinate along the thinnest part of the ring. Therefore we can conclude that in a ring with finite width the one-dimensional vortex is transformed into the usual two-dimensional vortex.

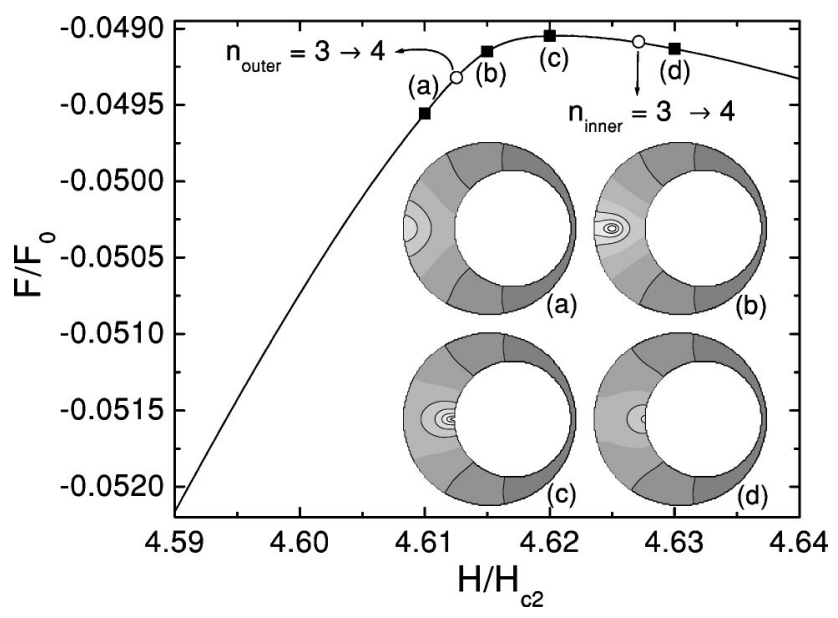

FIG. 6. The same as Fig. 5 but now for the last maximum in Fig. 4. In the inset a contour plot of the order parameter is shown for different values of the magnetic field which are given by the squares in the main figure. $L_{\text {outer }}$ is the vorticity as calculated along the outer perimeter of the ring and $L_{\text {inner }}$ is the vorticity calculated along the perimeter of the hole. 


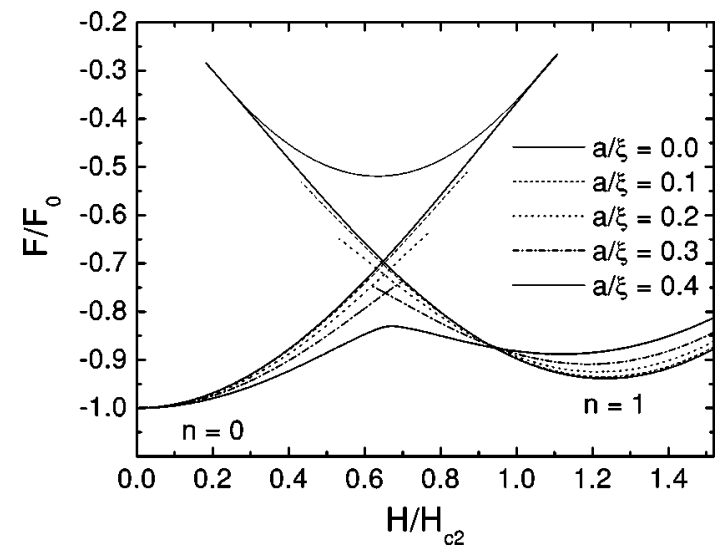

FIG. 7. The Gibbs free energy $F(H)$ at low magnetic fields for different displacements $a$ of the hole from the center of the ring. The thin solid curve (for $a=0$ ) corresponds to the saddle-point state.

The process of the appearance of a stable vortex in a ring with finite width is similar to the creation of a phase slip in wires of finite width in the presence of a transport current. In the latter case the distribution of the gauge-invariant momentum is not exactly uniform over the wire width. The maximum value of $p$ is obtained at the edges and as a result the order parameter vanishes first in these points. When a phase slip is created the distribution of the order parameter is not uniform over the width. With decreasing wire width this nonuniformity decreases but it will be uniform, strictly speaking, only in the limit $w \rightarrow 0$. With increasing wire width the phase slip transforms to the ordinary process of vortex/antivortex pair nucleating at the edges, penetrating deep into the superconductor and annihilating. In nonuniform rings the distribution of $p$ over the width is nonuniform and in contrast to wires with transport current it is nonsymmetric with respect to the ring width. Suppression of the order parameter first occurs only on the external side (when increasing the magnetic field) or on the internal side (when decreasing the magnetic field). As in the case of an one-dimensional ring we may call this state a stable ("frozen") phase slip state if the width of the ring, where the vortex penetrates, is less than $\xi$.

The ODV state of a nonuniform ring is very similar to the saddle-point state as found in Ref. 15 in case of a uniform ring of finite width and in Ref. 16 for the case of a disk. Note that for a uniform ring with zero width the nonuniform solution Eq. (5) corresponds to a saddle point of $F(\psi)$. In Fig. 7 the gradual decrease of the hysteresis with increasing displacement $a$ of the hole from the center of the ring is shown. It is seen that with increasing $a$ the region where metastable states exist decreases and ultimately vanishes for some critical displacement $a_{c}$. The saddle-point state (only shown for $a=0$, by the thin full curve) approaches the stable phase slip state when $a=a_{c}$. It corresponds to the boundary point of existence of ODV in the phase diagram of normalsuperconducting phase (the point $P_{2}$ in the notations of Ref. $3)$.

In Ref. 17 also nonuniform superconducting rings of finite width were investigated (but with larger ring radius). It was found that for low magnetic fields $F(H)$ is irreversible but for high magnetic fields the dependence $F(H)$ was reversible. In high fields the transition from a state with vorticity one to another state occurred through the same scenario as discussed above. A similar state was also considered in Refs. 18 and 19 in the framework of the linearized GinzburgLandau equations for a nonuniform ring of finite width. They found that in such a system the vortex may be stable in some magnetic-field region. In Ref. 19 analytical results for the magnetic field ranges where the vortex enter through the thinner part of the ring and through the thicker part were obtained. Our calculations generalize this result to lower temperatures (i.e., where the nonlinear Ginzburg-Landau equations must be used) and with inclusion of the nonzero demagnetization factor of the ring. We found the dependence of the Gibbs free energy (and magnetization) of this sample on the applied magnetic field. Our results also allowed us to compare the results of the one-dimensional model with the full two-dimensional one.

The ODV (or stable phase slip) state may be observed by magnetic experiments. Magnetic susceptibility is proportional $^{6}$ to $d j / d \Phi$ and the ODV state exhibits some characteristic peculiarities as was shown in the inset of Fig. 3. Magnetization $M$ is proportional to the current $j$ and hence $M(H)$ is reversible for samples where the ODV state exists (see Fig. 3 and the inset of Fig. 5). Furthermore, $M=0$ at $\Phi \simeq(n+1 / 2) \Phi_{0}$. Alternatively, because at $\Phi=(n+1 / 2) \Phi_{0}$ there is a point in the ring where the order parameter is equal to zero, this state may be found by transport measurements. For example, in Ref. 20 the dependence of the resistance of a hollow cylinder with radius of order $\xi$ was studied at temperature $T<T_{c}$ far from $T_{c}$. At $\Phi=\Phi_{0} / 2$ the resistance $(R)$ exhibited a maximum but the value of $R$ was a factor 3 less than the normal-state resistance $R_{n}$. If the cylinder has a nonuniform thickness the ODV state can be realized (because there is a similarity between cylinders of small thickness and rings of small width) as a stable state when $\Phi$ $=\Phi_{0} / 2$ and it will lead to a resistive (but superconducting) state even for very small currents and naturally the resistance of such a state will be less than $R_{n}$ as was found by Liu et $a l^{20}$

In conclusion, we studied the nonuniform state in a superconducting ring in which the order parameter vanishes at one point. It was shown that this state is characterized by a jump in the phase of the order parameter by $\pi$ near the point $\psi$ $=0$. It allows, in correspondence with the ordinary twodimensional Abrikosov vortex, us to call such a state a onedimensional vortex. This state is unstable in a uniform ring. In the case of a nonuniform ring [with variations of the geometrical $(d, w)$ or physical parameters $(\xi, \lambda)$ along the ring] or for a ring with an attached wire, this state may be realized in practice. For rings with nonzero width the ODV state transforms into the usual two-dimensional vortex. We also showed that this state is the remnant of the saddle-point state connecting two superconducting states with different vorticity as found in a uniform ring. The latter state becomes stable in a nonuniform ring. 
The work was supported by the Flemish Science Foundation (FWO-Vl), the "Onderzoeksraad van de Universiteit Antwerpen," the "Interuniversity Poles of Attraction Program-Belgian State, Prime Minister's Office-Federal Office for Scientific, Technical and Cultural Affairs," and the European ESF-Vortex Matter. One of us (D.Y.V.) was supported by FWO-Vl and partially by RFBR Grant No. N01-02-16593. Discussions with Professor A. Geim and comments from Professor J. Berger are gratefully acknowledged.
*Electronic address: peeters@uia.ua.ac.be

${ }^{1}$ J. Berger and J. Rubinstein, Phys. Rev. Lett. 75, 320 (1995).

${ }^{2}$ E.M. Horane, J.I. Castro, G.C. Buscaglia, and A. Lopez, Phys. Rev. B 53, 9296 (1996).

${ }^{3}$ J. Berger and J. Rubinstein, Phys. Rev. B 56, 5124 (1997).

${ }^{4}$ M. Tinkham, Phys. Rev. 129, 2413 (1963).

${ }^{5}$ J.S. Langer and V. Ambegaokar, Phys. Rev. 164, 498 (1967).

${ }^{6}$ X. Zhang and J. Price, Phys. Rev. B 55, 3128 (1997).

${ }^{7}$ A. Bezryadin, A. Buzdin, and B. Pannetier, Phys. Rev. B 51, 3718 (1995).

${ }^{8}$ Numerical analysis shows that for large rings $L \gg \pi$ the solution $t(s)=t_{1}-\left(t_{1}-t_{0}\right) / \cosh ^{2}\left[s \sqrt{\left(t_{1}-t_{0}\right) / 2}\right]$ found in Ref. 5 works very well for arbitrary currents $j<j_{c}$. The reason is that for rings with $R \gg 1$ the "energy" $E$ is close to $E_{\max }$ and the roots $t_{1}$ and $t_{2}$ practically coincide. As a result the situation is analogous to the one considered in Ref. 5.

${ }^{9}$ This result is a generalization of a result obtained in Ref. 5 for the saddle-point of Eq. (2) for the case of an infinite long wire with vanishing transport current.

${ }^{10}$ H.J. Fink and V. Grunfeld, Phys. Rev. B 33, 6088 (1986).

${ }^{11}$ H.J. Fink and V. Grunfeld, Phys. Rev. B 31, 600 (1985).

${ }^{12}$ H.J. Fink, Phys. Rev. B 45, 4799 (1992); 48, 3579 (1993).
${ }^{13}$ C. Ammann, P. Erdös, and S.B. Haley, Phys. Rev. B 51, 11739 (1995).

${ }^{14}$ It is interesting to note that the results shown in Fig. 2 correspond qualitatively to the time evolution of the order parameter for a uniform ring in the case where the vorticity increases with one unit. The only difference is that for a uniform ring the current density, generally speaking, is not equal to zero at the point where the order parameter vanishes. The reason is that for timedependent processes the full current density is equal to the sum of the superconducting $j_{s}$ and the normal $j_{n}$ parts. In the point $f=0, j_{s}=0$ but $j_{n} \neq 0$.

${ }^{15}$ B.J. Baelus, F.M. Peeters, and V.A. Schweigert, Phys. Rev. B 63, 144517 (2001)

${ }^{16}$ V.A. Schweigert and F.M. Peeters, Phys. Rev. Lett. 83, 2409 (1999).

${ }^{17}$ B.J. Baelus, F.M. Peeters, and V.A. Schweigert, Phys. Rev. B 61, 9734 (2000).

${ }^{18}$ J. Berger and J. Rubinstein, Phys. Rev. B 59, 8896 (1999).

${ }^{19}$ J. Berger, Connectivity and Superconductivity, edited by J. Berger and J. Rubenstein (Springer, New York, 2000), p. 138.

${ }^{20}$ Y. Liu, Yu. Zadorozhny, M.M. Rosario, B.Y. Rock, P.T. Carrigan, and H. Wang, Science 294, 2332 (2001). 